\title{
Récepteur Multi-normes pour les Réseaux de Capteurs de l'loT médical
}

\section{Multi-standard transceiver for the Internet of Medical Things}

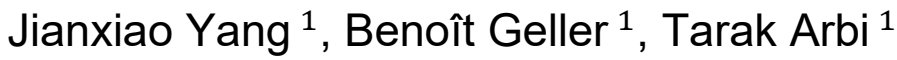 \\ ${ }^{1}$ Unité d'Informatique et d'Ingénierie des Systèmes U2IS, École Nationale Supérieure de Techniques Avancées Ensta \\ ParisTech, jianxiao.yang, benoit.geller, tarak.arbi @ensta-paristech.fr
}

RÉSUMÉ. Dans cet article, nous proposons une conception originale de réception pour le mode bande étroite de la couche physique de la norme d'loT médical IEEE 802.15.6, compatible avec un récepteur WiFi. Elle inclut la synchronisation de trame, la synchronisation en fréquence et la synchronisation temporelle. Les résultats de simulations montrent que les performances du système proposé sont très proches des performances théoriques.

ABSTRACT. In this paper, an original physical layer design of the IEEE 802.15.6 narrowband receiver is proposed, including frame synchronization, timing synchronization, and carrier frequency synchronization. This proposed singlecarrier system can be incorporated into a conventional multi-carrier system of the IEEE 802.11 transceiver by reusing its hardware resource. Simulation results show that the performances of the proposed system approach closely the theoretical performances.

MOTS-CLÉS. Wireless Body-Area Network (WBAN), Internet des Objets, Couche Physique, IEEE 802.15.6, Synchronisation de la trame, Synchronisation en Fréquence, Synchronisation Temporelle, IEEE 802.11, OFDM.

KEYWORDS. Wireless Body-Area Network (WBAN), Internet of Things (IoT), Physical Layer Design, IEEE 802.15.6, Frame Synchronization, Timing Synchronization, Carrier Frequency Synchronization, IEEE 802.11, OFDM.

\section{Introduction}

La norme IEEE 802.15.6 [IEE 12a] est dédiée aux communications sans fil, à très faible consommation énergétique et à faible couverture (portée de $3 \mathrm{~m}$ ) opérante à proximité du corps humain pour offrir en temps réel des services médicaux fiables tels que la mesure de paramètres vitaux et des services non médicaux comme les services de divertissement.

Contrairement au mode UWB de la norme 802.15.6, le mode bande étroite [LEE 09] aspire à devenir la norme de référence de l'internet des objets (IoT) pour l'industrie médicale. C'est pour cette raison, et différemment des travaux [LEE 09], [CHO 10], [MAT 15], [PRI 14] et [LIA 13], que cet article se concentre sur ce mode. Néanmoins, le mode bande étroite de la norme IEEE 802.15.6 doit coexister dans la bande ISM des $2.4 \mathrm{GHz}$ avec des concurrents de taille tels que notamment IEEE 802.11a/b/g/n (WiFi) [IEE 12b], IEEE 802.15.1 (Bluetooth) [IEE 05a], IEEE 802.15.4 (ZigBee) [IEE $05 \mathrm{~b}]$.

Pour favoriser l'essor du mode bande étroite de la norme IEEE 802.15.6, nous proposons un récepteur OFDM à l'instar de [Mic 01] (à multiples sous-porteuses orthogonales) pouvant par exemple être utilisé par un récepteur WiFi classique, et qui servirait alors de récepteur multi-standards.

Cet article est organisé comme suit. Dans la section suivante, nous décrivons les caractéristiques des différentes normes WiFi 802.11a/n/ac. La section 3 rappelle les spécificités du signal de la norme WBAN. La section 4 détaille la conception de la couche physique proposée pour la norme 802.15.6 en mode bande étroite. La section 5 discute les résultats de simulations. Enfin, diverses conclusions sont données à la fin de cet article. 


\section{Caractéristiques des normes IEEE $802.11 \mathrm{a} / \mathrm{b} / \mathrm{g} / \mathrm{h} / \mathrm{ac}$}

La norme IEEE 802.11 a initialement défini trois types de couches physiques FHSS, DSSS et IR. Ce standard n'est pas resté figé. En fait, au cours des années, de nombreuses révisions ont été apportées par les normes $802.11 \mathrm{a} / \mathrm{b} / \mathrm{g} / \mathrm{n} / \mathrm{ac}$. Le tableau ci-dessous résume les caractéristiques des premières normes apparues chronologiquement.

\begin{tabular}{|c|c|c|c|c|}
\hline Normes & Transmission & Bande de fréquence & Débit maximum & Modulation \\
\hline $\mathbf{8 0 2 . 1 1 a}$ & OFDM & $5 \mathrm{GHz}$ & $54 \mathrm{Mbits} / \mathrm{s}$ & $\begin{array}{c}\text { DBPSK, DQPSK } \\
16-\mathrm{Q} A M, 64-\mathrm{Q} A M\end{array}$ \\
\hline $\mathbf{8 0 2 . 1 1 b}$ & DSSS & $2.4 \mathrm{GHz}$ & $11 \mathrm{Mbits} / \mathrm{s}$ & DBPSK, DQPSK \\
\hline $\mathbf{8 0 2 . 1 1 g}$ & OFDM & & & \\
\hline
\end{tabular}

Tableau 2.1. Caractéristiques des normes $802.11 \mathrm{a} / \mathrm{b} / \mathrm{g}$

La norme IEEE 802.11n améliore les normes 802.11a/g en utilisant le système MIMO (entrées multiples, sorties multiples) pour offrir un débit qui peut en théorie atteindre $540 \mathrm{Mbits} / \mathrm{s}$. La norme IEEE 802.11ac améliore davantage le débit et facilite la transmission pour de multiples utilisateurs. Cette norme supporte deux bandes différentes de fréquences, à savoir $2.4 \mathrm{GHz}$ et $5 \mathrm{GHz}$.

Toutes les trames des standards $802.11 \mathrm{a} / \mathrm{g} / \mathrm{n} / \mathrm{ac}$ basées sur l'OFDM sont composées des champs suivants:

\begin{tabular}{|c|c|c|c|c|c|c|c|}
\hline & $10 \times 0.8$ us & $2.5 \times 3.2$ us & 4 us & & & & PSDU \\
\hline $802.11 \mathrm{a} / \mathrm{n} / \mathrm{ac}$ & L-STF & L-LTF & L-SIG & X-STF & X-LTF & X-SIG & MAC \\
\hline
\end{tabular}

Figure 2.1. Structure de la trame de $802.11 \mathrm{a} / \mathrm{n} / \mathrm{ac}$

Il y a une partie de préambule dans les normes 802.11a, comprenant les champs L-STF (LegacyShort Training Field), et L-LTF (Legacy-Long Training Field). Pour les normes plus avancées comme $802.11 \mathrm{n} / \mathrm{ac}$ qui utilise la technologie MIMO, il y a aussi une seconde partie de préambule, comprenant X-STF et X-LTF, où la lettre X représente HT (High Throughput) pour 802.11n ou VHT (Very High Throughput) pour $802.11 \mathrm{ac}$. La première partie du préambule dite «legacy » differencie la norme 802.11 des autres normes dans la même bande et signe le début de toutes les générations $802.11 \mathrm{a} / \mathrm{n} / \mathrm{ac}$. L-STF contient 10 répétitions d'une séquence courte de $0.8 \mu \mathrm{s}$ pour que les récepteurs puissent retrouver l'en-tête, contrôler le gain (AGC-Automatic Gain Control) et estimer le décalage en fréquence (CFO-Carrier Frequency Offset). Le champ L-LTF suivant contient 2.5 répétitions d'une séquence longue de $3.2 \mu$ s et permet les fonctions comme la synchronisation fine de trame et 1'estimation du canal. Le champ L-SIG (Legacy Signalling) contient des informations relatives aux 
paramètres de la modulation et du codage des données de 802.11 a. Pour $802.11 \mathrm{n} / \mathrm{ac}$, le champ X-SIG contient des informations spécifiques de modulation et de codage des données.

\section{Caractéristiques du mode bande étroite de la norme IEEE 802.15.6}

La figure 3.1. présente la structure de la trame de la norme IEEE 802.15.6. Elle est composée de 3 champs à savoir: le préambule PLCP (Physical Layer Convergence Protocol préambule), l'en-tête PLCP (PLCP Header) et le champ PSDU (Physical layer Service Data Unit). Ces deux derniers champs forment ce qui est appelé le PPDU (Physical Layer Protocol Data Unit).

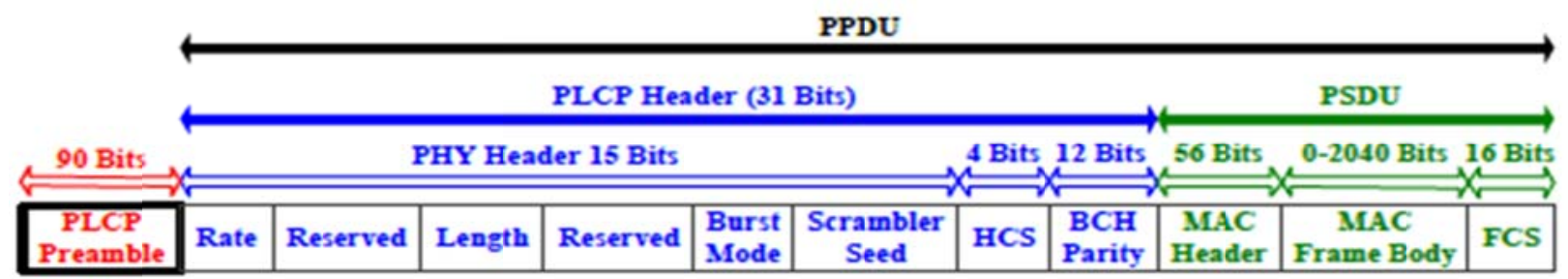

Figure 3.1. Structure de la trame WBAN

Le premier champ du préambule PLCP est une séquence d'apprentissage connue par le récepteur nécessaire pour plusieurs traitements en bande de base tels que la synchronisation de la trame, la synchronisation temporelle et la synchronisation fréquentielle. Pour le mode bande étroite, deux préambules sont définis afin de réduire les fausses alarmes causées par l'utilisation de canaux adjacents. Chaque séquence est obtenue en concaténant 63 bits d'une séquence pseudo-aléatoire avec les 27 bits suivants : 010101010101101101101101101 . Ce champ utilise la modulation $\pi / 2$-DBPSK et contient exactement 90 symboles complexes. Le deuxième champ d'en-tête PLCP comprend 31 bits et contient des informations relatives à la transmission telles que les paramètres de la modulation et du codage. Puisque ce champ utilise également la modulation $\pi / 2$-DBPSK, il contient 31 symboles complexes. Le dernier champ PSDU de la trame contient l'information utile. Pour le mode bande étroite, l'information binaire de ce champ est modulée par l'une des trois modulations différentielles suivantes : $\pi / 2$-DBPSK, $\pi / 4$-DQPSK ou $\pi / 8$-D8PSK.

Pour chacune de ces modulations, le flux binaire $b(n), n=0,1, \cdots, N-1$ est transformé en une séquence de symboles $s(k), k=0,1, \cdots,\left(N / \log _{2}(M)\right)-1$ comme suit :

$$
s(k)=s(k-1) \exp \left(j \varphi_{k}\right),
$$

où $M$ est l'ordre de la modulation et $s(-1)=\exp (j \pi / 2)$ est la référence pour le premier symbole du préambule. Les valeurs des transitions entre les symboles $\varphi_{k}$ sont donnés par le tableau 3.1, le tableau 3.2 et le tableau 3.3 pour les modulations $\pi / 2$-DBPSK, $\pi / 4$-DQPSK et $\pi / 8$-D8PSK respectivement.

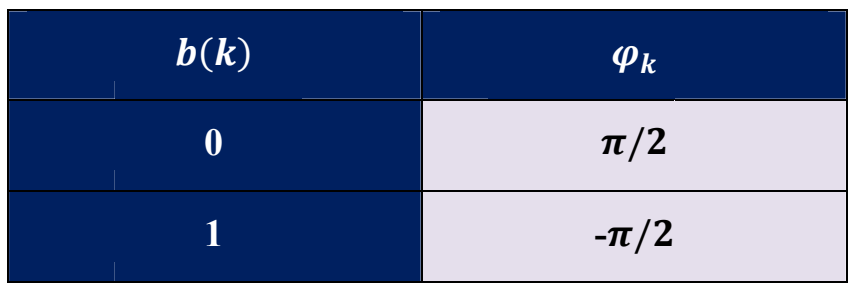

Tableau 3.1. Modulation $\pi / 2-D B P S K$ 


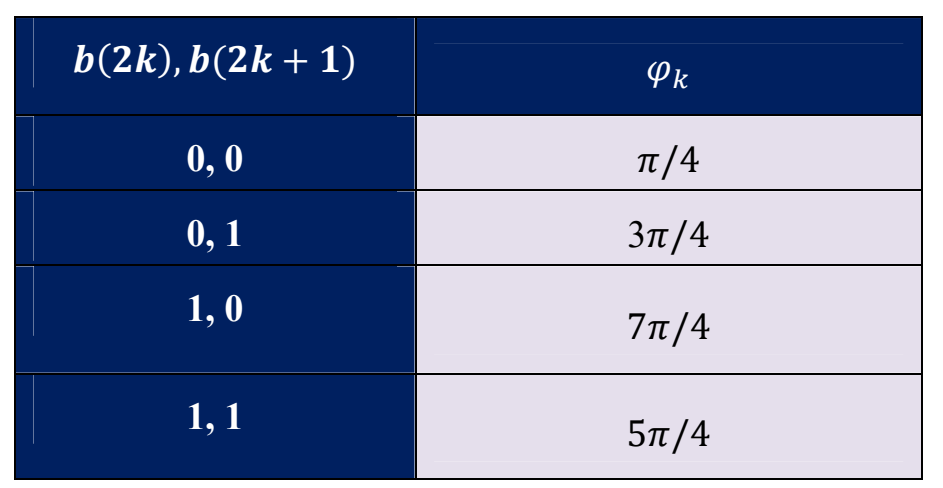

Tableau 3.2. Modulation $\pi / 4-D Q P S K$

\begin{tabular}{|c|c|}
\hline$b(2 k), b(2 k+1), b(2 k+3)$ & $\boldsymbol{\varphi}_{k}$ \\
\hline $\mathbf{0 , 0} \mathbf{0}$ & $\pi / 8$ \\
\hline $\mathbf{0 , 0 , 1}$ & $3 \pi / 8$ \\
\hline $\mathbf{0 , 1}, \mathbf{0}$ & $7 \pi / 8$ \\
\hline $\mathbf{0 , 1}, \mathbf{1}$ & $5 \pi / 8$ \\
\hline $\mathbf{1 , 0} \mathbf{0}$ & $15 \pi / 8$ \\
\hline $\mathbf{1 , 0} \mathbf{1}$ & $13 \pi / 8$ \\
\hline $\mathbf{1 , 1 , 0}$ & $9 \pi / 8$ \\
\hline $\mathbf{1 , 1} \mathbf{1}$ & $11 \pi / 8$ \\
\hline
\end{tabular}

Tableau 3.3. Modulation $\pi / 8-D 8 P S K$

À cause de la faible couverture et du faible débit de transmission, le canal peut être considéré comme un canal non sélectif en fréquence et à évanouissements lents. Par conséquent, le signal reçu peut-être modélisé par :

$r(t)=h \sum_{n} s_{n} u\left(t-n T_{s y m b}\right)+w(t)$,

où $u(t)$ est un filtre de mise en forme en racine de cosinus surélevé, $h$ est l'atténuation de canal qui peut être considérée constante pendant la transmission d'un paquet.

Les traitements du signal en bande de base du côté récepteur doivent inclure la synchronisation de trame, la synchronisation en fréquence et la synchronisation temporelle avant d'effectuer une détection classique de symboles. Il est à noter que la synchronisation de phase n'est pas effectuée grâce à la nature de la détection différentielle des symboles (cf. modulations précédentes).

\section{Conception de la couche physique}

Cette section détaille les algorithmes proposés pour les différents types de synchronisation : trame, puis en fréquence, et enfin en temps. Dans les normes $802.11 \mathrm{a} / \mathrm{n} / \mathrm{ac}$, les synchronisations peuvent être établies grâce aux champs L-STF et L-LTF. En général, la synchronisation de la trame est faite dans le domaine des fréquences. La synchronisation en fréquence est implémentée à la fois dans le domaine temporel et des fréquences. Enfin, comme la synchronisation temporelle peut être traitée comme un cas 
particulier d'égalisation, elle peut en fait être réalisée dans l'étape d'égalisation en fréquence. Si on peut utiliser le préambule en fréquence (par simple transformée de Fourier du préambule temporel), on peut réutiliser le hardware utilisé dans la norme IEEE 802.11 pour la synchronisation de la norme IEEE 802.15.6.

\subsection{Synchronisation de la trame}

Puisque le système IEEE 802.15.6 est un système de transmission par paquet, localiser le début du paquet (SOF) doit être la première tâche à réaliser en amont de tous les autres traitements en bande de base. Par conséquent, cette tâche doit tolérer plusieurs imperfections non encore traitées à ce stade telles qu'un mauvais échantillonnage, un décalage en fréquence et le bruit additif.

Afin de réaliser une synchronisation robuste de la trame en utilisant le signal reçu échantillonné avec une fréquence $f_{\text {samp }}=2 f_{\text {symb }}$, l'algorithme suivant est proposé:

$\hat{n}_{S O F}=\arg \left\{\max _{n}\left\{\sum_{l=1}^{L}\left\|\sum_{p=0}^{89-l} r\left(n+p+l \frac{T_{\text {symb }}}{T_{\text {samp }}}\right) r^{*}(n+p) * \prod_{q=0}^{l} \exp \left(-j \tilde{\varphi}_{p+q}\right)\right\|\right\}\right\}$,

où $\hat{n}_{S O F}$ est le début de la trame estimée, $r(n)$ est la n-ième observation du signal reçu, $T_{\text {symb }}=$ $1 / f_{\text {symb }}$ est la période symbole, $T_{\text {samp }}=1 / f_{\text {samp }}$ est la période d'échantillonnage, $\tilde{\varphi}_{n}$ est la transition de phase entre les symboles $s(n-1)$ et $s(n)$.

Il y a deux points à noter; tout d'abord, le terme de corrélation $r\left(n+l \frac{T_{\text {symb }}}{T_{\text {samp }}}\right) r^{*}(n)$ réduit l'impact du décalage en fréquence; de plus, l'indice $l$ indique que la corrélation n'est pas uniquement évaluée entre les symboles consécutifs mais qu'elle est évaluée également entre des symboles espacés dans le temps, ce qui augmente la robustesse de l'algorithme.

Nous proposons de prendre $L=2$; ceci s'avère suffisant pour localiser le début de la trame avec un décalage en fréquence (CFO) $f_{C F O}=0.1 f_{\text {symb }}$ et un rapport signal sur bruit (SNR) $S N R=-1 d B$.

\subsection{Synchronisation en fréquence}

La synchronisation en fréquence est la deuxième tâche à effectuer. Elle a un impact important sur la synchronisation temporelle et la détection finale des symboles. Notre algorithme de synchronisation, développé pour compenser le décalage en fréquence entre l'émetteur et le récepteur, se décompose en deux étapes; la première concerne une synchronisation grossière en fréquence, tandis que la deuxième traite de la synchronisation fine en fréquence.

La synchronisation grossière en fréquence est effectuée comme suit :

1. Transformer chaque $N_{F F T}^{C F O}$ observations de signal reçu dans le domaine des fréquences par FFT. Ces observations dans le domaine des fréquences sont désignées par $\widetilde{R}(k), \mathrm{k}=0,1, \ldots, N_{F F T}^{C F O}-1$;

2. Transformer les $N_{F F T}^{C F O} / 2$ symboles du préambule dans le domaine des fréquences par FFT. Ces observations dans le domaine des fréquences sont désignées par $\widetilde{P}(k), \mathrm{k}=0,1, \ldots, N_{F F T}^{C F O} / 2-1$;

3. Localiser le maximum de corrélation entre les deux séquences dans le domaine des fréquences, comme suit :

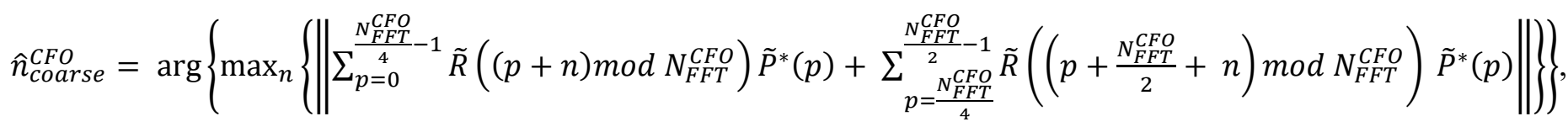


où $\bmod N_{F F T}^{C F O}$ se réfère à l'opération modulo $N_{F F T}^{C F O}$.

La valeur estimée du décalage en fréquence est :

$$
\hat{f}_{\text {coarse }}^{C F O}=\hat{n}_{\text {coarse }}^{C F O} \frac{f_{\text {samp }}^{\text {sam }}}{N_{F F T}^{C F O}}
$$

4. Compenser le décalage en fréquence précédent $\hat{f}_{\text {coarse }}^{C F O}$.

Il est à noter que la valeur estimée par la synchronisation grossière a une précision de l'ordre de $f_{\text {samp }} / N_{F F T}^{C F O}$. La taille de la FFT détermine donc la précision de l'algorithme : plus la taille de la FFT est importante, meilleure est la précision de l'algorithme. Nous proposons de prendre $N_{F F T}^{C F O}=128$. Par conséquent, pour chaque paquet contenant 90 symboles de préambule, il y a deux valeurs estimées partiellement corrélées.

Après la compensation de la valeur estimée par l'algorithme de la synchronisation grossière du décalage en fréquence, une synchronisation fine en fréquence est effectuée comme suit :

$$
\hat{f}_{\text {fine }}^{C F O}=\frac{1}{2 \pi L T_{\text {symb }}} * \tan ^{-1}\left(\sum_{l=1}^{L} \frac{1}{(90-l) l} \sum_{p=0}^{89-l} \tilde{r}(n+l) \tilde{r}^{*}(p) \prod_{q=0}^{l} \exp \left(-j \tilde{\varphi}_{p+q}\right)\right) .
$$

Afin de réduire la complexité du système, l'estimation fine du décalage en fréquence est effectuée comme suit :

$$
\hat{f}_{\text {fine }}^{\text {CFO }}=\frac{1}{\pi T_{\text {symb }}} \tan ^{-1}\left(\hat{V}_{\text {fine }}^{\text {CFO }}\right)
$$

où $\widehat{V}_{\text {fine }}^{C F O}$ est obtenu par un simple filtre à réponse impulsionnelle infinies (IIR) du premier ordre :

$$
\widehat{V}_{\text {fine }}^{C F O}=(1-\alpha) \widehat{V}_{\text {fine }}^{C F O}+\frac{\alpha}{l} \tilde{r}(p+l) * \tilde{r}^{*}(p) \prod_{q=0}^{l} \exp \left(-j \tilde{\varphi}_{p+q}\right) .
$$

Dans les simulations de la section 5, nous proposons de prendre $\mathrm{L}=2$ et $\alpha=0.01$.

\subsection{Synchronisation Temporelle}

L'objectif de cette tâche est d'estimer le délai $\tau$ du signal, où :

$$
r(t-\tau)=h \sum_{n} s_{n} u\left(t-n T_{s y m b}-\tau\right)+w(t-\tau) .
$$

De façon différente de la synchronisation temporelle conventionnelle, constituée généralement de deux blocs à savoir (Symbol Interpolator) et (Timing Error Detector TED), l'algorithme proposé tend à estimer la réponse impulsionnelle globale du canal $h u\left(t-n T_{s y m b}-\tau\right)$. Par conséquent, la synchronisation temporelle comprend deux blocs : estimation de canal et égalisation de canal.

L'estimateur de canal a pour objectif d'estimer la fonction de transfert en fréquence du canal comme suit :

1. Transformer chaque $N_{F F T}^{T R}$ observations de signal reçu dans le domaine des fréquences par FFT. Ces observations dans le domaine des fréquences sont désignées par $\tilde{R}(k), k=0,1, \ldots, N_{F F T}^{T R}-1$.

2. Insérer 1 zéro entre chaque couple de symboles du préambule. Ensuite, transformer chaque $N_{F F T}^{T R}$ points de la séquence obtenue dans le domaine des fréquences par FFT ; les observations dans le domaine des fréquences sont désignées par $\tilde{P}(k), k=0,1, \ldots, N_{F F T}^{T R}-1$. 
3. Comme pour l'estimateur de canal conventionnel du système OFDM, la réponse en fréquence du canal peut être estimée par : $\widehat{H}(k)=\tilde{R}(k) / \tilde{P}(k)$.

4. Interpoler linéairement la réponse en fréquence du canal entre chaque couple de valeurs consécutives de $\widehat{H}(k)$. On obtient donc, une nouvelle fonction de transfert de canal $\widehat{H}_{I}(k)$ ayant $2 N_{F F T}^{T R}$ points.

Ensuite l'égaliseur de canal déclenche la méthode overlap-save (OLS) [PRO 07] afin d'effectuer l'égalisation en fréquence des observations du signal reçu suréchantillonné après compensation fréquentielle. Il doit :

1. Prendre les $N_{F F T}^{T R}$ observations du bloc OLS précédent.

2. Prendre les $N_{F F T}^{T R}$ nouvelles observations à égaliser.

3. Transformer les $2 N_{F F T}^{T R}$ observations dans le domaine des fréquences, les points correspondants sont désignés par $R(k)$.

4. Effectuer l'égalisation : $\widehat{D}(k)=R(k) / \widehat{H}_{I}(k)$.

5. Transformer les échantillons de $\widehat{D}(k)$ dans le domaine temporel.

6. Extraire les derniers $N_{F F T}^{T R}$ points échantillonnés. Le premier de chaque couple d'échantillons permet d'obtenir le symbole DMPSK. Par comparaison avec les symboles émis, ceci permet de mesurer les taux d'erreur binaire (BER) du paragraphe suivant.

Nous proposons de prendre $N_{F F T}^{T R}=64$.

\section{Résultats de simulation}

Dans cette section, les performances du système proposé pour les modulations $\pi / 2$-DBPSK (voir figure 5.1), $\pi / 4$-DQPSK (voir figure 5.2) et $\pi / 8$-D8PSK (voir figure 5.3) sont évaluées en terme de taux d'erreur binaire BER pour deux scénarios : le premier est la simulation avec un décalage temporel aléatoire $\tau \in\left[-T_{\text {symb }} / 2, T_{\text {symb }} / 2\right]$ (voir les courbes indiquées par 'RTO sim') ; l'autre scénario ne comprend pas uniquement un décalage temporel aléatoire, mais également un décalage en fréquence important $f_{C F O}=0.1 f_{\text {symb }}$ (voir les courbes indiquées par 'RTO $+10 \%$ CFO sim'). Dans ces simulations, tous les blocs précédents de traitements en bande de base sont activés (synchronisation de la trame, synchronisation en fréquence et synchronisation temporelle). Les meilleures performances que l'on peut obtenir sur un canal à bruit blanc additif gaussien sont les performances théoriques (voir courbes indiquées par 'theory') [XIO 06] [PRO 08] qui sont obtenues en supposant une parfaite synchronisation entre l'émetteur et le récepteur. Ces courbes théoriques nous servent donc de référence absolue pour comparer les résultats de notre proposition.

Les figures 5.1. 5.2. et 5.3. montrent que les courbes de taux d'erreur (BER) du système proposé restent très proches des performances théoriques optimales avec uniquement un décalage temporel aléatoire RTO. On remarque également que dans des conditions très difficiles de synchronisation en considérant une dégradation supplémentaire avec un décalage en fréquence important, les algorithmes proposés restent robustes pour les modulations $\pi / 2$-DBPSK et $\pi / 4$-DQPSK. 


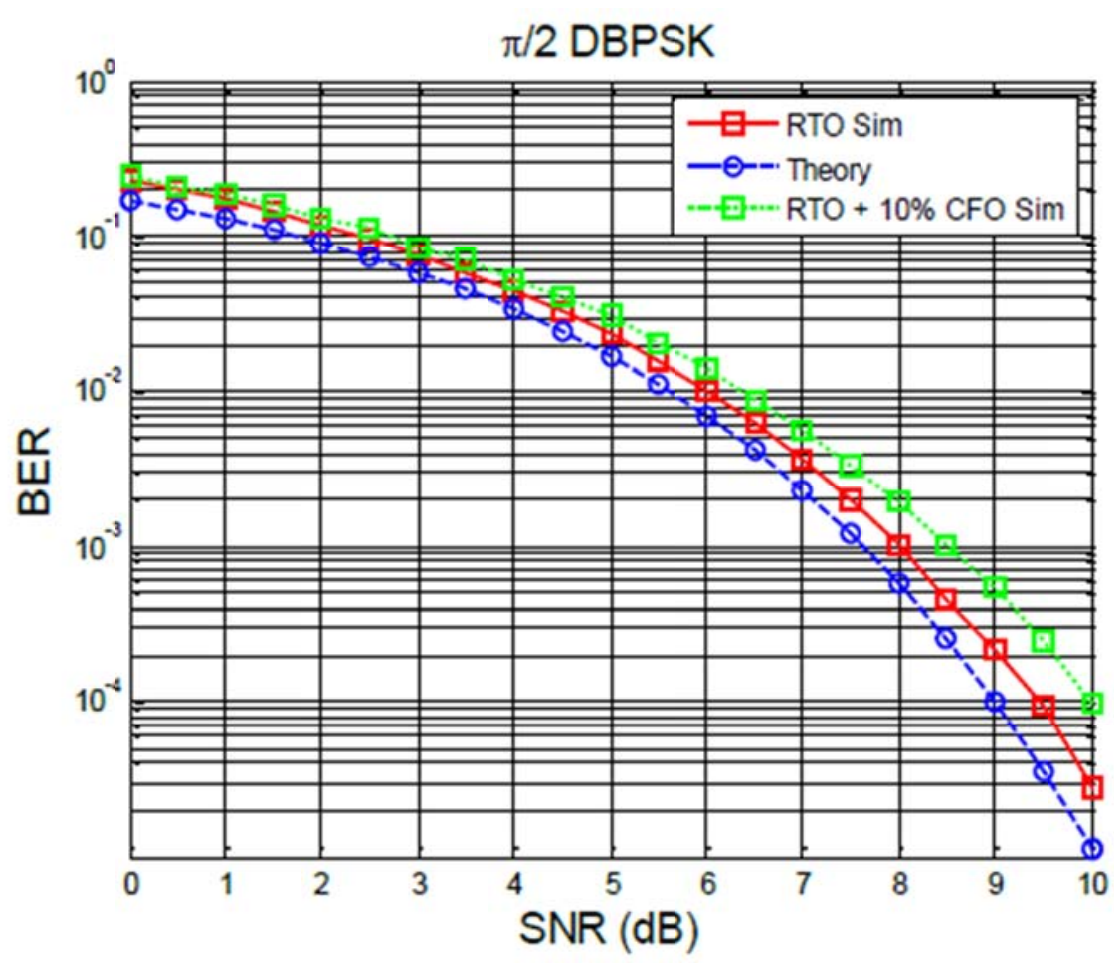

Figure 5.1. Performance du système proposé pour la modulation $\pi / 2-D B P S K$

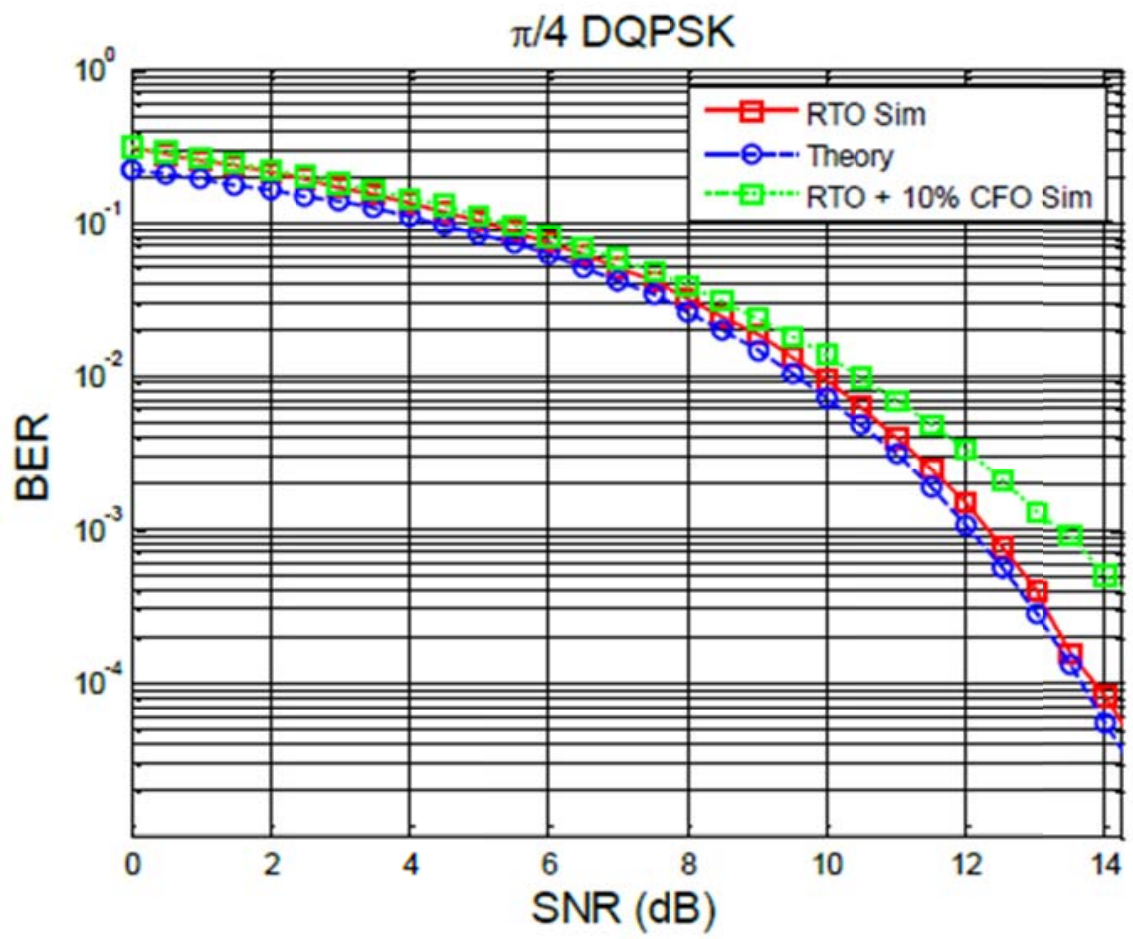

Figure 5.2. Performance du système proposé pour la modulation $\pi / 4-D Q P S K$ 


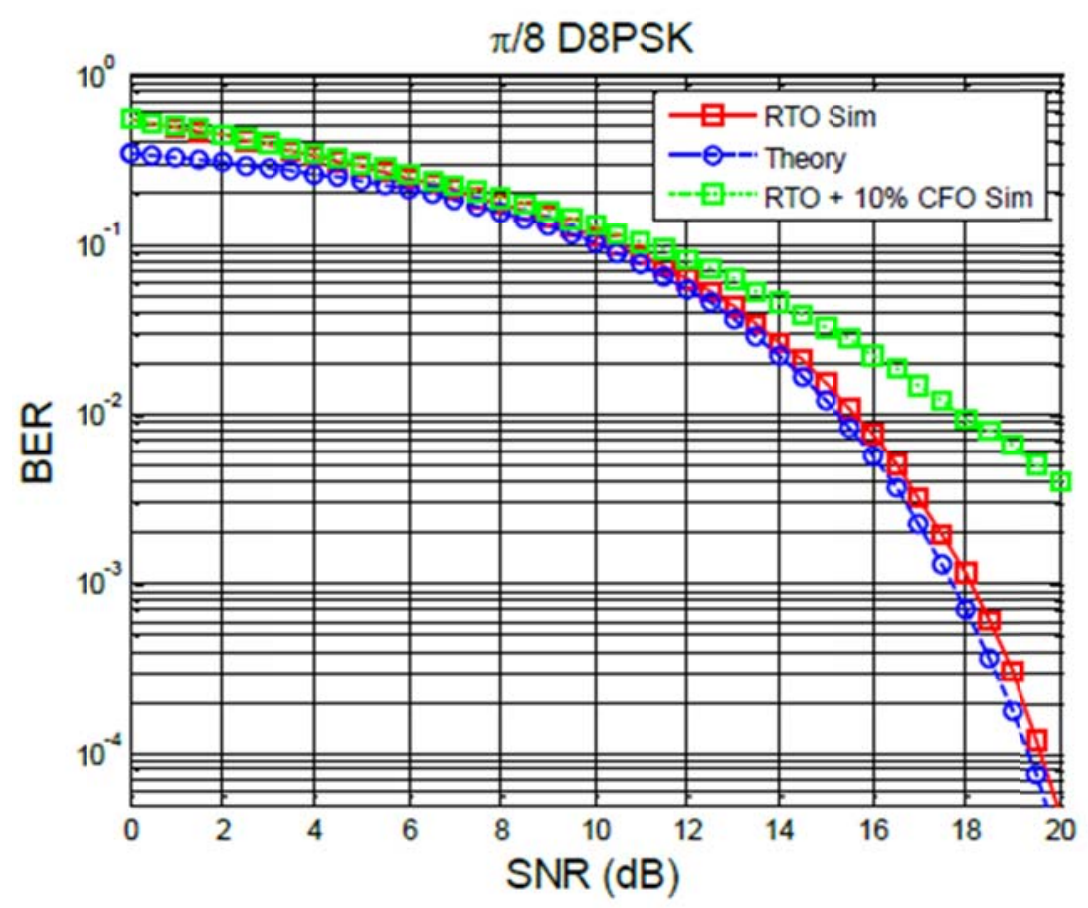

Figure 5.3. Performance du système proposé pour la modulation $\pi / 8-D 8 P S K$

\section{Conclusion}

Dans cet article, une conception des algorithmes de la couche physique pour le mode bande étroite de la norme IEEE 802.15.6 est proposée. Contrairement à la conception conventionnelle de la couche physique de récepteur mono-porteuse, les algorithmes de traitement du signal proposés peuvent être directement utilisés par un système OFDM tel que dans le WiFi. Les résultats de simulations montrent que les performances des algorithmes proposés sont très proches des performances théoriques avec une parfaite synchronisation sur un canal gaussien avec un décalage temporel aléatoire, ce qui rend cette conception attractive pour la conception d'un récepteur multi-normes et multi-modes. Dans la suite de notre étude, nous voulons inclure dans notre récepteur multi-standards, un décodeur souple [VAN 08] et un design cross-layer pouvant servir à la localisation.

\section{Bibliographie}

[IEE 12a] IEEE Standard for local and metropolitan area network part 15.6: Wireless Area Body Network, 2012.

[LEE 09] Lee C., Kim J., Lee H. S. and Kim J., «Physical layer designs for WBAN systems in IEEE 802.15.6 proposals», in the proceedings of $9^{\text {th }}$ International Symposium on Communications and Information technology, 2009. ISCIT 2009. Pp. 841-844, Icheon, 2009.

[PRI 14] Priya Mathew, Lismi Augustine, Deepak Kushwaha, Vivian D. and David Selvakumar., «Hardware Implementation of NB PHY Baseband Transceiver for IEEE 802.15.6 WBAN, » International Conference on Medical Imaging, m-Health and Emerging Communication Systems (MedCom 2014), Nov. 2014.

[LIA 13] Liang Y., Zhou Y. and. Lu Y., «The design and implementation of IEEE 802.15.6 Baseband on FPGA» in the proceedings of International Conference on Health Informatics, IFMBE Proceedings 42, Nov. 2013

[CHO 10] Choi B., Kim B., Lee S., Wang K., Kim Y. and Chung D., «Narrowband Physical Layer Design for WBAN System», in the proceedings of First International Conference on Pervasive Computing Signal Processing and Application (PCSPA), 2010, pp. 154-157, Harbin, 2010.

[MAT 15] Mathew P., Augustine L., Kushwaha D., Desalphine V. and Selvakumar A. D., «Implementation of NB PHY transceiver of IEEE 802.15.6 WBAN on FPGA», in the proceedings of 2015 International Conference on VLSI Systems, Architecture, Technology and Applications (VLSI-SATA), pp. 1-6., Bangalore, 2015. 
[IEE 12b] IEEE Standard for local and metropolitan area networks Part 11: Wireless LAN Medium Access Control (MAC) and Physical Layer (PHY) Specification, 2012.

[IEE 05a] IEEE Standard for local and metropolitan area networks part15.1: Wireless Medium Access Control (MAC) and Physical Layer (PHY) Specification for Wireless Personal Networks (WPANs), 2005.

[IEE 05b] IEEE Standard for Local and metropolitan area networks Part 15.4: Low-Rate Wireless Personal Area Networks (WPANs), 2005.

[MIC 01] Micheal S., Stefan F., Gunnar F. and Heinrich Meyr, « Optimum Receiver Design for OFDM-Based Broadband Transmission»IEEE Transaction on communications, vol. 49, no. 4, April 2001.

[PRO 07] Proakis J. G., and Manolakis, D. K., « Digital Signal Processing», 4th Edition., Pearson, 2007.

[XIO 06] Xiong F., Digital Modulation Techniques (Second Edition), Artech House, April, 2006.

[PRO08] Proakis J.G, Salehi M. «Digital communications» 5th ed. Mc Graw Hill, 2008.

[VAN 08] Vanstraceele C., Geller B., Barbot J.P., Brossier J.M., " A Low Complexity Block Turbo Decoder Architecture », IEEE Transactions on Communications, vol. 56, no 12, pp. 1985-1989, Dec. 2008. 